\title{
RNA interference on chromosomes
}

\author{
R Kelly Dawe \\ Transcriptional silencing in fission yeast requires several core components of the RNA interference machinery. A new \\ study suggests that the recently discovered RNA-induced initiation of transcriptional gene silencing complex binds \\ stably to silent chromatin, where it recruits short interfering RNAs and destroys nascent RNA molecules in cis.
}

Whether in animals, plants or fungi, the discovery of epigenetic gene silencing (heritable changes in gene expression without changes in DNA sequence) is a fascinating story. Like many other discoveries in genetics, it began as an obscure observation in an unlikely organism (the petunia) and gradually worked its way up to model organisms as powerful as Schizosaccharomyces pombe $e^{1,2}$. The post-transcriptional form of epigenetic silencing is known as RNA interference (RNAi). The key RNAi proteins are Dicer (Dcr1), an enzyme that cleaves double-stranded RNA (dsRNA) into small fragments known as short interfering RNAs (siRNAs); RNA-dependent RNA polymerase (Rdp1), which uses siRNAs to polymerize additional dsRNA; and RNAinduced silencing complex (RISC), which mediates the degradation of RNAs homologous to RISC-associated siRNAs. In contrast, transcriptional silencing occurs at the chromatin level. Silent chromatin is almost always associated with a methylated form of histone H3 (H3-Lys9) and a conserved H3-Lys9 binding protein, Swi6.

For many years, these two forms of silencing were viewed as separate and distinct. But links between transcriptional and post-transcriptional silencing began to appear in the late 1990s (ref. 3), and in 2002, two key discoveries in S. pombe made the connection irrefutable $e^{4,5}$. These studies showed that without Dcr1, Rdp1 or the presumed RNase component ${ }^{6}$ of RISC known as Argonaute (Agol), pericentromeric heterochromatin was abolished ${ }^{4}$ and a transcriptionally inactive mating type locus (mat)

R. Kelly Dawe is in the Department of Plant Biology and Department of Genetics, University of Georgia, Athens, Georgia 30602, USA. e-mail: kelly@plantbio.uga.edu could no longer be silenced ${ }^{5}$. A search for proteins mediating the interaction between RNAi and transcriptional silencing led to the identification of the RNA-induced initiation of transcriptional gene silencing (RITS) complex, which contains Agol, a heterochromatinbinding protein known as Chp1, Tas3 and siRNAs ${ }^{7}$. On page 1174 of this issue, Noma et $a l .{ }^{8}$ present convincing evidence that RITS is an integral component of the epigenetic silencing pathway in S. pombe.

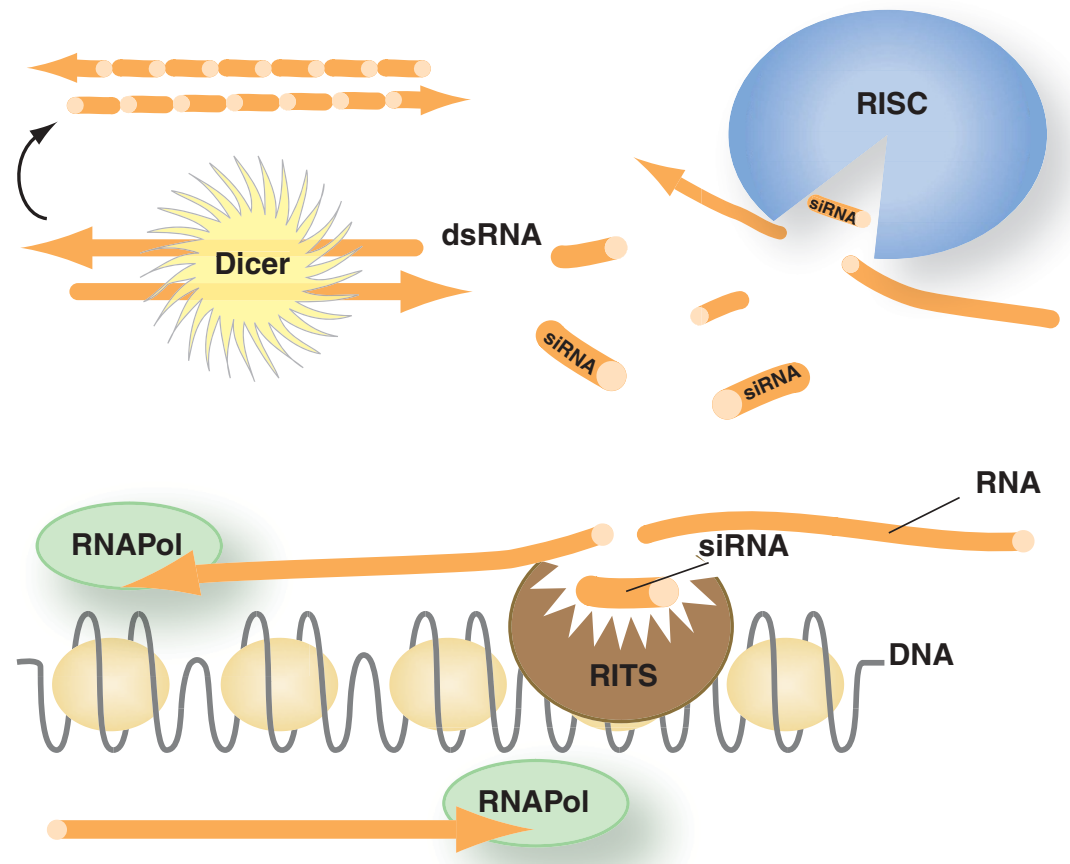

Figure 1 A simplified view of how RITS fits into the RNAi pathway. RNAi is initiated by transcription from both strands of a DNA sequence. The resulting dsRNA is degraded by Dcr 1 to produce siRNAs. Both RISC and RITS use siRNAs and Ago 1 to cleave RNA. RISC cleaves RNA in trans, whereas RITS binds to chromatin and is thought to destroy newly produced RNAs in cis. 
mat region. The spreading reaction is mediated in part by Swi6 (ref. 5), and Noma et al. ${ }^{8}$ now show that it involves RITS as well.

Their interpretations are based on several observations. First, they found that a mutation in the chromodomain of Chp1 abolishes RITS binding to chromatin. They also found a loss of RITS binding in $c l r 4^{-}$mutants, which lack methylated H3-Lys9 binding sites for RITS. In addition, they found that RITS spreads in a Swi6-dependent manner. These data support the view that Swi6 mediates the recruitment of $\mathrm{Clr} 4$, which provides the necessary H3-Lys9 binding sites for RITS. The authors note that siRNAs and Dcrl are not required for spreading or subsequent maintenance of RITS at heterochromatic loci. Instead, the Chp1 chromodomain is primarily responsible for the affinity of RITS for silent chromatin. The sequence-independence of this spreading reaction presumably allows RITS to exert control over sequences incapable of initiating the RNAi response.

\section{Enforcing the silence}

Drawing on the analogy of RNAi as a genetic immune response ${ }^{9}$, several authors have hypothesized that RNAi might guide chromatin-silencing complexes back to the gene that produced the dsRNA ${ }^{3-5,7}$. Further, if components of the RNAi machinery remain bound to transcriptionally silenced loci, rare transcripts that escape silencing could be rec- ognized and destroyed before transcription is completed. In a key set of experiments, Noma et al. show that the free, chromatin-unbound form of RITS does not associated with siRNAs (with the caveat that small quantities of siRNAs might have been missed). When bound to chromatin through H3-Lys9, however, RITS seems to become especially receptive to siRNAs. RITS may acquire its siRNAs from post-transcriptional (trans-acting) RNAi activity or more locally from aberrant RNAs processed at the source of transcription. Once bound to chromatin and primed with siRNAs, RITS probably becomes the center of localized RNA destruction, ensuring that stray transcripts from silent chromatin are cleaved in cis (presumably by Ago1) $)^{6}$. Thus, although siRNAs are not necessarily required to target RITS to specific chromatin sites, siRNAs and RITS both seem to be involved in the predicted cis-acting RNAi function (Fig. 1).

\section{Initiating events}

The authors also discuss briefly the issue of what factors might be involved in the earliest events of Dcr1-dependent, sequence-specific heterochromatin formation. It is important to incorporate Dcrl and siRNAs into any model for the initiation of silencing, because the genetic data for this at pericentromeres and other loci and quite good ${ }^{4,10}$. Although silencing at mat is genetically more complex than at pericentromeres, there are good indications that the two silent domains are regulated in a similar manner. Aside from the facts that pericentromeres and mat contain nearly identical sequences and bind the same set of proteins ${ }^{4,5}$, a dcr $1^{-}$swi $^{-}$double mutant fails to initiate and maintain mat silencing ${ }^{8}$. Given that swi $6^{-}$alone does not have this effect $^{5}$, siRNAs are probably required in some form to designate mat as a silent domain.

The discovery that RITS binds efficiently to chromatin without siRNAs ${ }^{8}$ puts a damper on early predictions that RITS might serve as the primary initiator of chromatin silencing. An alternate possibility is that a larger silencing complex of some sort ${ }^{8}$ interacts with Clr4 (ref. 10). However this question is ultimately resolved, it's a good bet that the answer will come from $S$. pombe, where the combination of genetics and biochemistry has been very productive.

1. Baulcombe, D. Nature 431, 356-363 (2004).

2. Lippman, Z. \& Martienssen, R. Nature 431, 364-370 (2004).

3. Matzke, M., Matzke, A.J. \& Kooter, J.M. Science 293, 1080-1083 (2001).

4. Volpe, T.A. et al. Science 297, 1833-1837 (2002).

5. Hall, I.M. et al. Science 297, 2232-2237 (2002).

6. Song, J.J., Smith, S.K., Hannon, G.J. \& Joshua-Tor, L. Science 305, 1434-1437 (2004).

7. Verdel, A. et al. Science 303, 672-676 (2004).

8. Noma, K. et al. Nat. Genet. 36, 1174-1180 (2004).

9. Plasterk, R.H. Science 296, 1263-1265 (2002).

10. Schramke, V. \& Allshire, R. Science 301, 1069-1074 (2003).

\title{
A new gene on the $\mathrm{X}$ involved in Fanconi anemia
}

\section{Nazneen Rahman \& Alan Ashworth}

\author{
A new study shows that Fanconi anemia complementation group $B$ is caused by mutations in a previously \\ uncharacterized gene located on the $\mathrm{X}$ chromosome. Its unique chromosomal localization identifies FANCB as a \\ potential weak link in a key DNA-repair and tumor-suppressor pathway.
}

There are few better examples of the proposition that studying rare genetic disorders can illuminate important biological pathways than research into Fanconi anemia, which has identified several genes important for DNA repair. Fanconi anemia is characterized by congenital

Nazneen Rahman is in the Section of Cancer Genetics, The Institute of Cancer Research, Cotswold Rd, Sutton, Surrey SM2 5NG, UK. e-mail:nazneen.rahman@icr.ac.uk.Alan Ashworth is at The Breakthrough Breast Cancer Research Centre, The Institute of Cancer Research, Fulham Rd, London SW3 6JB, UK. e-mail:alan.ashworth@icr.ac.uk. defects, progressive bone marrow failure and cancer susceptibility ${ }^{1}$. Its distinctive cellular phenotype involves chromosomal instability and hypersensitivity to DNA cross-linking agents. Eleven distinct complementation groups for Fanconi anemia have been defined using somatic cell fusion techniques, and the genes underlying eight of these complementation groups have been identified. Now, Meetei et al. $^{2}$ on page 1219 describe an X-linked gene defective in individuals with Fanconi anemia complementation group B (FA-B). Because of its unique chromosomal location, the gene encoding this newly discovered component of the Fanconi anemia complex may represent a particularly vulnerable component of the DNA repair pathway responsible for maintaining genomic integrity.

Six of the proteins involved in Fanconi anemia (FANCA, FANCC, FANCE, FANCF, FANCG and FANCL) are part of a protein complex that functions as a ubiquitin ligase, of which FANCL is probably the catalytic subunit $^{3}$. In response to DNA damage or in S phase of the cell cycle, this complex catalyzes the monoubiquitination of FANCD2 (Fig. 1). A defect in any one of these core proteins results in failure to monoubiquitinate FANCD2, emphasizing the importance of this modification. But exactly how this complex 